\title{
Review: exercise therapy may reduce pain and disability in osteoarthritis of the hip or knee
}

Van Baar ME, Assendelft WJ, Dekker J, et al. Effectiveness of exercise therapy in patients with osteoarthritis of the hip or

knee. A systematic review of randomized clinical trials. Arthritis Rheum 1999 Jul;42:1361-9.

QUESTION: In patients with osteoarthritis of the hip or knee, does exercise therapy

reduce pain and disability?

\section{Data sources}

Studies were identified by searching Medline (1966 to September 1997), EMBASE/Excerpta Medica (1988 to September 1997), CINAHL (1982 to September 1997), the Cochrane Controlled Trials Register, and bibliographies of relevant articles.

\section{Study selection}

Studies were selected if they were full reports, published in any language, of randomised controlled trials (RCTs) involving patients with osteoarthritis of the hip or knee that was clinically or radiologically assessed; treatment included exercise therapy; and $\geq 1$ of the outcome measures included pain, self reported or observed disability, or patient's global assessment of effect. Studies were excluded if they involved perioperative exercise therapy or if the intervention groups received identical exercise therapy.

\section{Data extraction}

2 reviewers independently assessed the quality of study methods, and 1 reviewer extracted data on patients, interventions, outcomes, and adverse effects. Effect sizes were calculated for outcomes.

\section{Main results}

10 published RCTs and 1 RCT accepted for publication met the inclusion criteria. The mean number of patients in each group ranged from 7 to 146. 4 RCTs compared different exercise therapy programmes, and 8 compared exercise therapy with no treatment or with interventions that did not involve exercise therapy. Of the 2 RCTs with acceptable validity and sufficient power, 1 studied both aerobic and resistance exercise. In both of these RCTs, exercise therapy led to an improvement in pain (effect sizes $0.58,95 \%$ CI 0.54 to 0.62 [study 1 ]; 0.47 , CI 0.44 to 0.50 , for aerobic exercise; and 0.31 , CI 0.28 to 0.34 , for resistance exercise [study 2]); self reported disability (effect sizes 0.26 , CI 0.22 to 0.30 [study 1]; 0.41, CI 0.38 to 0.44 , for aerobic exercise; and 0.36 , CI 0.33 to 0.39 , for resistance exercise [study 2]); and observed disability in walking (effect sizes 0.28 , CI 0.24 to 0.32 [study 1]; 0.89, CI 0.85 to 0.93 , for aerobic exercise; and 0.31 , CI 0.28 to 0.34 , for resistance exercise [study 2]). 1 study with acceptable validity and sufficient power showed a benefit for exercise therapy for patients' global assessment of effect (effect size 0.64, CI 0.60 to 0.68). 1 RCT with acceptable validity and low power also showed a benefit of exercise therapy for pain (effect size 0.20 , CI 0.08 to 0.32 ) and patients' global assessment of effect (effect size 1.40 , CI 1.28 to 1.52 ). However, a worsening of walking disability (effect size -0.11 , CI -0.17 to -0.05 ) was seen in this RCT. The evidence did not show superiority for any particular type of exercise therapy programme.

\section{Conclusion}

In patients with osteoarthritis of the hip or knee, exercise therapy may reduce pain and disability.

\section{COMMENTARY}

The systematic review by van Baar and colleagues examines the possible benefit of exercise therapy in patients with osteoarthritis of the hip or knee. The methods used by the authors meet the current standards for systematic reviews. As the authors appropriately point out, however, the methods of most of the included clinical trials are not well done. Only 1 trial, which was written by 1 of the review authors, reached $75 \%$ of the maximal score for the validity criteria, and all but 3 trials had validity scores $\leq 50 \%$ of the optimum. Therefore, although the authors of the review have produced a work that approaches the ideal, their conclusions should be sharply weighted by the quality of study material. On the basis of this review, there is some equivocation about the use of exercise therapy in patients who have osteoarthritis of the hip or knee. In fact, little information is provided in the evaluated trials about the extent of osteoarthritis for which such an intervention might be useful. Further, as mentioned by the authors, several types of interventions have been recorded in the published trials, including aerobic exercises, resistance exercise, weight bearing exercise, and walking programmes.

For patients with osteoarthritis, exercise therapy may reduce pain and disability, but it still must be determined what type of exercise routine should be offered and for which patients such programmes are applicable.

Jean Y Reginster, MD, PhD WHO Collaborating Center and University of Liège Liège, Belgium 